\title{
Links between Autonomic Dysfunction and Metabolic Syndrome
}

\section{Federica Giampetruzzi and Gabriella Garruti*}

Section of Internal Medicine, Endocrinology, Andrology and Metabolic Diseases, Department of Emergency and Organ Transplantation (DETO), University of Bari "Aldo Moro", School of Medicine, Bari, Italy

\begin{abstract}
The autonomic nervous system (ANS) plays a key role in the control of a number of vital functions including cardiovascular, endocrine/neurovascular, gastrointestinal, genitourinary, pupil and thermoregulatory functions. Its abnormalities have been associated with early mortality, sudden death, silent myocardial infarction, gastrointestinal diseases.

The manifestation of the ANS dysfunction in several human diseases is underestimated. Evidences exist on the important role of ANS dysfunctions in different clinically relevant conditions, including diabetes mellitus, chronic functional constipation, scleroderma, thalassemia major.

Beside the classical evaluation in patients with diabetes mellitus, little is known about the effects of metabolic factors on ANS dysfunction. The metabolic syndrome (MetS) includes a cluster of frequent abnormalities (impaired fasting glycaemia, dyslipidemia, arterial hypertension and increased visceral adiposity) predisposing to the atherosclerotic changes and increased cardiovascular mortality. Early signs of autonomic dysfunction are often found in subjects with MetS even in the absence of diabetes. Epidemiological studies demonstrated that diabetics display a cardiovascular risk which is twice that of sex- and age-matched non-diabetic population. Manifestations of such a high cardiovascular risk of subjects with DM are the frequent silent myocardial infarctions (MI)s of diabetics which are often due to impaired cardiovascular autonomic function. Only recently major attention has been given to the interactions between impaired glucose tolerance (IGT) and cardiovascular autonomic dysfunctions. When increased waist circumference (one of the features of the MetS) and IGT are both present, cardiovascular autonomic dysfunction also occurs. Some adipokines (e.g. adiponectin) seem to play a role in cardiovascular risk and autonomic dysfunction. This review will therefore focus on some subtle aspects linking ANS dysfunction and MetS.
\end{abstract}

Keywords: Cardiovascular; Metabolic syndrome; Autonomic neuropathy; Adipokines

List of Abbreviations: ANS: Autonomic Nervous System; CAF: Cardiovascular Autonomic Function; CAN: Cardiovascular Autonomic Neuropathy; CT: Cough Test; CVD: Cardiovascular Disease; DB: Deep Breathing; DCCT: The Diabetes Control and Complications Trial; DM: Diabetes Mellitus; EDIC: Epidemiology of Diabetes Interventions and Complications; IGT: Impaired Glucose Tolerance; HRV: Heart Rate Variability; LS: Lying to Standing; MI: Myocardial Infarction; MetS: Metabolic Syndrome; OSAS: Obstructive Sleep Apnea Syndrome; PH: Postural Hypotension; PNS: Parasympathetic Nervous System; SNS: Sympathetic Nervous System; SST: Sweat Spot Test; VS: Valsalva Manouvre

\section{Introduction}

Epidemiological studies unequivocally show that subjects with metabolic syndrome (MetS) as well as subjects with Diabetes Mellitus (DM) are at increased risk of cardiovascular diseases (CVD).

Subjects displaying a cluster of factors predisposing to the Atherosclerotic Cardiovascular Disease might be included in the syndrome named Syndrome X [1-3], (Table 1) and show a risk for stroke and coronary heart disease which is threefold higher as compared with that of controls [4]. Diabetics display a risk for CVD which is twice that of sex- and age-matched non-diabetic population and they frequently experience silent myocardial infarctions (MI) $s[5,6]$. Clinically unrecognized MIs might be the consequence of an impaired cardiovascular autonomic function which finally evolves to an overt cardiovascular autonomic neuropathy (CAN).

As far as the links between DM and MetS are concerned, subjects with Type $2 \mathrm{DM}$ obligatory have one of the diagnostic criteria of MetS (glycaemia $\geq 100 \mathrm{mg} / \mathrm{dl}$ ), but they do not always show the other diagnostic features for the MetS. However, in subjects with DM, cardiovascular risk becomes higher when clinical features of the MetS are present along with DM $[7,8]$. When an impaired balance between the sympathetic and parasympathetic regulation of the cardiovascular function arises, a worsening of the prognosis of Diabetes already occurs.

In this review we will report recent updates on the link between autonomic dysfunction and the presence of MetS with respect to screening tests, and coexistence of several metabolic abnormalities.

\section{Screening Tests for Autonomic Dysfunction}

In the Rochester Diabetic Neuropathy study no correlation was often found between autonomic symptoms and autonomic cardiovascular tests in subjects with Type 2 Diabetes $[9,10]$. Therefore an analysis of cardiovascular reflexes with tests which are sensitive, and non-invasive represent the only way to confirm the diabetic CAN. The cardiovascular reflexes are automatic responses in which heart and vascular functions are modified by stimulating different receptors involved in the control of heart rate, circulating blood volume, blood vessel diameter (vasodilation and vasoconstriction).

*Corresponding author: Gabriella Garruti, MD PhD, Assistant Professor, Section of Internal Medicine, Endocrinology, Andrology and Metabolic Diseases, Department of Emergency and Organ Transplantation (DETO), University of Bari “Aldo Moro", Policlinico, Piazza G. Cesare 1170124 Bari, Italy, Tel: 0039328/5497739; E-mail: gabriella.garruti@uniba.it

Received December 19, 2015; Accepted January 08, 2016; Published January 15, 2016

Citation: Giampetruzzi F, Garruti G (2016) Links between Autonomic Dysfunction and Metabolic Syndrome. J Metabolic Synd 5: 195. doi:10.4172/2167 0943.1000195

Copyright: $\odot 2016$ Giampetruzzi F, et al. This is an open-access article distributed under the terms of the Creative Commons Attribution License, which permits unrestricted use, distribution, and reproduction in any medium, provided the original author and source are credited. 


\section{Central obesity (necessary)}

Plus any two of the following conditions:

2. Elevated triglycerides $(>150 \mathrm{mg} / \mathrm{dl})$

3. Decreased HDL cholesterol ( $<40 \mathrm{mg} / \mathrm{dl}$ in males: $<50 \mathrm{mg} / \mathrm{dl}$ in females)

4. Elevated arterial blood pressure $(>130 / 85 \mathrm{mmHg})$

5. Elevated fasting blood glucose $(>100 \mathrm{mg} / \mathrm{dl})$

Table 1: Diagnostic criteria for the metabolic syndrome according to International Diabetes Federation.

\begin{tabular}{|c|c|c|c|}
\hline Test & PNS & SNS & Both PNS/SNS \\
\hline CT & + & & \\
\hline DB & + & & \\
\hline HRV & & & + \\
\hline LS & + & & \\
\hline PH & & + & \\
\hline SST & + & & + \\
\hline VS & & & + \\
\hline
\end{tabular}

Abbreviations: CT: Cough Test; DB: Deep Breathing; HRV: Heart Rate Variability LS: Lying To Standing; PH: Postural Hypotension; PNS: Parasympathetic Nervous System; SNS: Sympathetic Nervous System; SST: Sweat Spot Test; VS: Valsalva Manouvre;

Table 2: Sympathetic and parasympathetic screening tests.

The tests based on cardiovascular reflexes include Beat-to-beat heart rate variation (DB), heart rate changes after standing (LS), heart rate response to Valsalva maneuver (Vs), heart rate changes induced by cough (cough test, CT), systolic blood pressure response to upright position (PH) [11,6,12-14], (Table 2). All tests are usually performed with portable computerized systems that are used for step-by-step performance of several cardiovascular tests for autonomic neuropathy. Tests are performed after an overnight fast but never after overnight hypoglycaemia. It was unequivocally demonstrated that hypoglycaemia blunts vagal baroreflex sensitivity and sympathetic response to hypotension.

Before each test, subjects are instructed to refrain from smoking and drinking coffee for at least $8 \mathrm{~h}$, to lying in the supine position for at least 30 minutes and a basal ECG is registered.

For DB, a parasympathetic function test, a $1 \mathrm{~min}-\mathrm{ECG}$ is performed when the subject is lying supine and deeply breathes 6 times per minute. The expiration/inspiration R-R ratio is calculated and compared with that found in age-matched control subjects.

For LS, a parasympathetic function test, the patient is invited to stand suddenly and the R-R interval is measured at beats 15 and 30 after standing and the $30 / 15$ ratio is calculated.

For VS, the patient exhales for 15 min into the mouthpiece of a manometer exerting a pressure of $40 \mathrm{mmHg}$ and the ratio between the longest and the shortest R-R interval is measured.

For $\mathrm{PH}$ assessment, supine systolic blood pressure is measured after the patient is lying down for $30 \mathrm{~min}$ and orthostatic blood pressure after the patient is suddenly standing for 2 minutes. Orthostatic hypotension is diagnosed when the fall in systolic blood pressure levels is $\geq 30 \mathrm{mmHg}$ or that of diastolic $\mathrm{BP}$ fall was $\geq 10 \mathrm{mmHg}$ in response to the postural change from the supine to the upright position [15]. Orthostatic hypotension is known to reflect sympathetic dysfunction [16] (Table 2).

$\mathrm{CT}$, a parasympathetic test function, evaluates the cough-mediated increase in heart rate. During the test the patient is in the supine position, ECG is performed when patient breathed for 15 seconds (basal) and again when he coughed 3 times. The $\mathrm{R}-\mathrm{R}$ ratio between the shortest R-R interval after the last cough and the mean R-R interval during regular respiration is calculated [13]. For each test the range of normal values is changing with age.

Another method to screen autonomic dysfunctions is to analyze heart rate variability [17]. It is measured in the resting position either over a period or for 24 hours. The time-domain and frequency-domain indices of heart rate variability are usually analyzed and power spectral analysis in the low frequency spectrum $(0.05-0.15 \mathrm{~Hz})$ and in the high frequency spectrum $(0.15-0.5 \mathrm{~Hz})$ and then the low frequency/ high frequency ratio might be calculated by specific software. In normal subjects the LF components prevail during the day while the components of the HF spectrum are predominant during the night. The explanation is that the sympathetic activity (responsible for the low frequency components), is mainly present during the day, while the vagal activity is predominant during night [7].

Sympathetic skin response (SSR) and Quantitative pupillography have been also recently used to screen autonomic fuction in children and adults, as elsewhere reported [18-22].

\section{Impaired Autonomic Function in Metabolic Syndrome and Diabetes}

Because of CAN, diabetics might not only experience silent myocardial infarctions but also silent hypoglycaemia and a high ASA risk during major surgery. Autonomic nervous system is anatomically poorly accessible and few direct physiological tests are available to study cardiovascular autonomic function (CAF). Therefore, some indirect clinical tests are used as screening tests which detect impaired CAF on the basis of heart responses to a simple stimulus [23]. In subjects with abnormal screening tests, the diagnosis might be completed with more sensitive techniques, but indirect screening tests help to select candidate subjects for more sophisticated analyses [23]. The diagnosis of CAN is usually made on the basis of the criteria of Ewing recently revised by Spallone et al. [17], but often when two tests are already impaired and the diagnosis of CAN is made might be too late to reverse the prognosis. Viceversa, sometimes early parasympathetic neuropathy may improve. Of note, in a longitudinal study [24], Gottsäter et al. demonstrated that after 7-10 years a subgroup of patients who were diagnosed with parasympathetic neuropathy, did not fulfil the criteria for the diagnosis anymore because of improved metabolic control. Therefore in a recent study, we thought to consider as an early deficit of CAF the detection of at least one pathological test. CAF was analysed by utilizing five different tests in a cohort of relatively young subjects with T2D. To each abnormal test we gave a score to establish a grading of severity of impaired CAF. In our cohort, the occurrence of 2 abnormal tests was rare, but the prevalence of at least one abnormal test was as high as $33.9 \%$. In two multicenter studies and a population study of type 2 diabetics, the prevalence of CAN was $16-22 \%[25,26]$, and slightly lower than the prevalence found in our study. A plausible explanation is that the authors used only 2 (i.e., DB, LS) or 3 (i.e., DB, LS. PH) screening tests, whereas in our cohort, 5 tests (i.e., DB, LS, $\mathrm{CT}, \mathrm{VS}, \mathrm{PH}$ ) were invariably performed in triplicate, likely increasing the sensitivity of tests. Concerning MetS in our young cohort, $65 \%$ subjects had MetS according to IDF, but the prevalence of MetS among subjects showing at least one abnormal test for CAF was more than $85 \%$. A significant positive correlation between impaired CAF and MetS was confirmed with two different models of multivariate analysis. It was previously assessed an association between parasympathetic dysfunction (pathologic cardiac response to DB) and some features of the MetS according to WHO [27]. However, our report demonstrates 
that, MetS according to the criteria of IDF, is associated with a higher occurrence of an early deficit of CAF in a relatively young cohort of type 2 diabetics. In the same cohort we also analyzed the possible associations between the single components of MetS and the detection of an early deficit of CAF.

We found a significant correlation between the occurrence of at least one pathologic test for CAF and overweight (BMI >25), which supports the negative role played by the excess of body fat on cardiovascular risk. Overweight also has a negative effect on glycaemic control. In this line of evidence in our study we demonstrate a significant association between high $\mathrm{HbAlc}$ values ( $\mathrm{HbAlc}>7.0 \%$ ) and the occurrence of at least one abnormal test [8]. HbAlc is an established parameter to assess mean glycemia over the preceding 3 months.

Many studies have already demonstrated that either an acute or a chronic poor glycaemic control might facilitate the appearance of CAN [28-30]. These data suggest a long term benefit of intensive therapy on microvascular complication explained by the treatment group differences in mean HbAlc levels over time. Moreover, in EDIC study patient with CAN showed an increase in left ventricular mass and massto-volume ratios compared with diabetics without CAN ( $\mathrm{p} 0.0001$ for each), changes consistent with left ventricular concentric remodeling that were independent of age, sex, and other traditional cardiovascular risk factors. From different meta-analysis the median value of mortality after 5 years was around $25 \%$ in diabetics with CAN and $4 \%$ in diabetics without CAN. If the diagnosis of CAN was based on the occurrence of 2 abnormal tests the relative risk of mortality was 3.5 [31,32]. Subjects from EDIC were recently analysed to test the association between testosterone levels and CAN, but "Testosterone levels" were not "associated with CAN among men with type1 diabetes" [33].

Interestingly, improving the glycaemic control also counteracts the early deficit of CAF or stops its progression (DCCT 1993). In studies utilizing heart rate variability as an index of CAF, mild CAF abnormalities improved if $\mathrm{HbAlc}$ values decreased from $9.5 \%$ to $8.4 \%$ $[34,35]$.

A strong association was found between the duration of Diabetes and CAN [36]. Both $\mathrm{PH}$ and decreased heart rate variability are more frequent and severe 5 years after the diagnosis of Diabetes [36]. Unexpectedly, we found no association between CAF score and the duration of Diabetes, however, the subjects of our cohort [8], probably had a better metabolic memory than that of subjects from previous studies, not only because they were younger than those considered in previous studies, but also because they experienced a program of education to healthy life-style together with insulin and /or oral antidiabetic agents of last generation since the onset of Diabetes.

Several factors associated with MetS might account for sympathetic over-activity. Excess of abdominal body (central obesity) is one of the obligatory component of MetS and increased central/visceral fat results in overproduction of inflammatory adipokines which also play a role in some pathogenic pathways inducing autonomic impaired balance and enhancing cardiovascular risk. The two hormones leptin and adiponectin (both originating from visceral adipose tissue), appear to be involved as well, since increased circulating levels of leptin and decreased circulating levels of adiponectin stimulate sympathetic overflow.

Several reports show that a higher cardiovascular risk is present in subjects displaying a cluster of factors predisposing to the Atherosclerotic Cardiovascular Disease and included in the syndrome named MetS(Table 1) [2,3]. Subjects with T2D always have one of the diagnostic criteria of (glycaemia $\geq 100 \mathrm{mg} / \mathrm{dl}$ ), but do not obligatory show other diagnostic features for MetS. Interleukin-6 is a multifunctional cytokine that plays a central role in inflammatory responses.

In both MetS and Diabetes C-reactive protein (CRP) and interleukin-6 (IL-6) play a pathogenetic role. C-reactive protein (CRP) and interleukin-6 (IL-6) are strongly interrelated since CRP is produced in the liver in response to IL- 6 in the acute-phase of inflammation. Both CRP and IL-6 are inflammatory markers and they have been found to be inversely related with reduced heart rate variability in a study concerning more than 200 male twins who had never had any symptom of coronary artery disease. Nonetheless, diabetic polyneuropathy correlates with CRP and IL-6 levels [36], the possibility exists that impaired autonomic function might induce chronic low-grade inflammation, but it has also been suggested that inflammation might induce cardiovascular dysfunction [36].

Early in the development of autonomic dysfunction, there was loss of heart rate variability (HRV), and this correlated with an increase in circulating markers of inflammation, such as C-reactive protein (CRP) and IL-6. Of great interest to us was the loss of HRV as well as the loss of sympathetic/parasympathetic balance, even before the advent of inflammation. Cardiac autonomic imbalance also correlated with markers of adipose tissue-derived inflammation (adiponectin-to-leptin ratio) and this was seen early in type 2 diabetes patients.

An increased sympathetic tone and decreased vagal response usually account for an impaired autonomic balance. In isolated adipocytes, an increased sympathetic tone is usually associated with inflammation since after $\beta$-adrenergic stimulation the levels of IL- 6 are increased [37]. In several studies increased levels of acetylcholine and/ or electrical stimulation of vagal nerve endings were associated with a reduced release of cytokines from inflammatory cells (macrophages) [38].

In central Obesity increased circulating leptin levels are present which might account for the activation of sympathetic nervous system found in MetS. Human leptin is a protein of 167 amino acids. It is mainly synthesized in the adipocytes of white adipose tissue, and there is a direct correlation between the total amount of fat in the body and the circulating levels of leptin $[39,40]$.

Very recently it was unequivocally demonstrated that Leptin responsive neurons exists in the nucleus of the solitary tract (NTS) which is one of the main regulatory sites of the sympathetic nervous system. Interestingly, the long form of the leptin receptor $(\mathrm{Ob}-\mathrm{Rb})$ has been detected in NTS of Sprague-Dawley rats and leptin injection in NTS elicits sympathoexicitatory responses through the stimulation of $\mathrm{Ob}-\mathrm{Rb}$ and mediate chemoreceptor afferent information to specific areas of the rostral ventrolateral medulla (RVLM) which are involved in the reflex control of arterial blood pressure [41]. Hyperleptinemia is associated with increased fat mass and human obesity. Interestingly, leptin is known to play a role in food intake regulation and energy balance at the level of several areas of the hypothalamus which are also involved in the switching on of the sympathetic nervous system. In animal models displaying leptin-resistance $(\mathrm{db} / \mathrm{db}$ mice), diabetes and obesity are associated with distraught circadian rhythm of blood pressure [42], To support the idea that leptin effects were mediated by the activation of the sympathetic nervous system there are studies demonstrating that intravenous infusion of leptin induces an increase in both arterial blood pressure and heart rate which is completely blunted by $\beta$-adrenergic blockers $[43,44]$. 
However, hyperleptinemia can be considered a marker of increased sympathetic tone and leptin insufficiency is associated with reduced sympathetic activity.

Not only low-grade inflammation but also hypo-adiponectinemia ensure in MetS and obesity. Adiponectin is a secretory protein uniquely encoded by adipocytes in different mammal species. Adipose tissue is richly innervated by both sympathetic and parasympathetic nerve endings and an impaired balance between the two autonomic branches has been claimed to influence adiponectin and cytokine secretion thus changing the inflammatory state of pathologic conditions characterized by visceral fat accumulation (e.g. metabolic syndrome, diabetes) [45].

Little is known about the effect of adiponectin in the regulation of sympathetic nervous system. However Adiponectin injection in the hypothalamic paraventricular nucleus depolarizes parvocellular neurons controlling both autonomic function and endocrine pathways [46]. Adiponectin plays an hypotensive effect either by modulating the NPY neurons of the NTS or stimulating the renal sympathetic nerve endings $[46,47]$. By contrast in conditions characterized by an increased sympathetic tone (e.g. cold exposure) serum adiponectin levels are suppressed [48]. In subjects with Type 2 Diabetes a direct correlation exists between increased vagal activity and adiponectin circulating levels [49].

Recent evidences suggest that a transient autonomic imbalance occurs before diabetes and/or overt autonomic neuropathy. New insights in the relationships between glucose tolerance and autonomic function have been recently pointed out. In subjects with impaired glucose tolerance (IGT), heart rate variability is already impaired. Heart rate analysis represents a non-invasive method to test cardiovascular autonomic function. However, some other studies demonstrate that in subjects with impaired glucose tolerance neither heart rate variability nor cardiovascular reflex tests (deep breathing, heart rate response to Valsalva maneuver, blood pressure response to standing up quickly) are sometimes able to discover a mild initial form of autonomic neuropathy which might be early detected with sympathetic skin response (SSR) that evaluates postganglionic sympathetic sudomotor function. In patients with IGT the amplitude of the SSR of two different limbs (right arm and leg) is often lower than that found in healthy subjects [50]. These data support the view that sympathetic sudomotor function might be impaired before cardiovascular autonomic function but unfortunately the above mentioned studies did not directly analysed the cardiovascular risk in subjects showing impaired SSR and it is possible that impaired SSR does not correlate with any other metabolic abnormality except for glucose tolerance.

In normalweight and normotensive individuals short-term hyperinsulinemia, which is one of the features of pre-diabetes, was already able to induce a decrease in heart rate variability [53]. Hyperinsulinemia is one of the features of pre-diabetes and the possibility exists that the early impairement of the cardiovascular reflex function occurring in both IGT and hyperinsulinemia/insulinresistance may be the cross-bridge between the increased cardiovascular disease risk and the early changes in glucose tolerance [53].

\section{Autonomic Imbalance associated with Cardiovascular Risk, Metabolic Syndrome and Obstructive Sleep Apnea Syndrome}

Subjects with MetS show an impaired function of the autonomic system. In different reports central obesity, insulin resistance and increased levels of high blood pressure which represent the features of the MetS, are accompanied by an increased sympathetic tone $[54,55]$.
Before overt diabetes, several subjects might experience prediabetes such as impaired glucose tolerance and hyperinsulinemia. In both conditions circadian regulation of cardiac autonomic function is compromised. In lean normotensive individuals [56], demonstrated that 2-day hyperinsulinemia reduced heart rate variability during night and blunted the nocturnal lowering of the arterial blood pressure.

It might be argued that the early impairement of the cardiovascular reflex function may represent the link between cardiovascular disease and early changes in glucose tolerance [55]. The cardiovascular reflex function includes a group of reflexes in which heart and circulatory functions changes in response to variations in heart rate, vascular tone, blood volume, or other variables.

Several studies demonstrate an increased activity of the sympathetic nervous system (SNS) in hypertension and insulin resistance which are components of the metabolic syndrome and/or Syndrome X. Central obesity is an obbligatory component of the MetS according to the International Diabetes Federation (IDF) and it is also associated with SNS hyperactivity. However, it is difficult to establish whether the impaired function of SNS is one of the causes of or one of the comorbidities of such a syndrome.

In a study involving more than 1000 subjects, an impaired function of the ANS seemed to be already present in subjects showing only one of the abnormalities of the MetS, even before the appearance of insulin resistance [56].

Therefore it is reasonable to believe that an impaired autonomic balance might be associated with the early negative changes of glucometabolic control and might play a potential role in switching on the pathogenic pathways which lastly produce diabetes [57].

Very often the excess of central fat is not only associated with diabetes and/or but also with obstructive sleep apnea syndrome (OSAS). This feature is clarly evident in subjects with severe forms of obesity undergoing bariatric surgery. The increased circulating levels of $\mathrm{CO} 2$ which are present in subjects with OSAS might be themselves responsible for an increased low-grade inflammatory state which favor sympathetic overactivity. To support the idea that fat excess is associated with autonomic dysfunction, Blüher's group recently demonstrated that overweight and obese children without diabetes or impaired glucose tolerance already showed a decrease in both parasympathetic and sympathetic activity.

\section{Lifestyle and Sympathetic Autonomic Dysfunction}

Few studies considered the dietary habits of subjects showing impaired cardiovascular autonomic function (CAF). In a recent paper from our group we screened for cardiovascular autonomic neuropathy 180 subjects with type 2 diabetes with mean age of 48 years and we also analyzed whether any relationship existed between the nutritional habits of our cohort and the occurrence of any deficit of the autonomic function [8]. Interestingly, subjects with impaired CAF more often had MetS as compared with subjects with normal CAF but also eat a higher amount of saturated fat (higher amount of cheese and fat meet) as compared with diabetics who did not show any sign of abnormal CAF and MetS Unexpectedly, subjects with impaired CAF and MetS chose a western-style diet even if they were living in a mediterranean area [8]. Some data already exist about subjects with MetS who showed an improvement in their chronic low-grade inflammatory state (reduction in serum concentrations of C-reactive protein, interleukin6, insulin-resistance, and improved endothelial function score) after experiencing a mediterranean-style diet (high content of whole grains, fruits, vegetables, nuts, and olive oil). This dietary model seems to be 
more effective than a balanced low-fat diet [58]. These data support the idea that lifestyle intervention program might reduce the risk of impaired autonomic function as already demonstrated by the Diabetes Prevention Program (DPP) where a low-glycaemic index and low-fat diet plus moderate exercise reduced the risk of autonomic neuropathy of one forth [59]. Regular exercise as well as dietary intervention program might also prevent autonomic dysfunction. In type 2 Diabetes, chronic aerobic activity (three-month exercise) restored heart rate variability, barorecptor sensitivity and vagal activity [60]. However exercise can be significantly increased only in early cardiac autonomic neuropathy, by contrast dietary intervention might be applied in any kind of autonomic dysfunctions and also in the presence of resting tachycardia, which is the extreme manifestation of severe autonomic neuropathy.

\section{Conclusion}

In conclusion, cardiovascular autonomic dysfunction is usually considered as a complication of diabetes, by contrast recent data point out its role at the very begininnig of the cascade of events inducing the chronic low-grade inflammation which represents the "primum movens" of both pre-diabetes and MetS. An emerging insight is the early impairement of autonomic function in childhood obesity in the absence either of overt diabetes or impaired glucose tolerance. However lifestyle intervention might improve and/or delay autonomic neuropathy and/or MetS either in childhood or adulthood.

\section{Acknowledgements}

The authors are grateful to Prof. Francesco Giorgino for precious suggestions.

\section{References}

1. Reaven GM (1993) Role of insulin resistance in human disease (syndrome $X$ ): an expanded definition. Annu Rev Med 44: 121-131.

2. Grundy SM, Cleeman JI, Daniels SR, Donato KA, Eckel RH, et al. (2005) American Heart Association; National Heart, Lung, and Blood Institute, 2005. Diagnosis and management of the Metabolic Syndrome: An American Heart Association/National Heart, Lung and Blood Institute Scientific Statement. Circulation 112: 2735-2752.

3. Zimmet P, Magliano D, Matsuzawa Y, Alberti G, Shaw J (2005) The metabolic syndrome: a global public health problem and a new definition. J Atheroscler Thromb 12: $295-300$

4. Isomaa B, Almgren P, Tuomi T, Forsén B, Lahti K, et al. (2001) Cardiovascula morbidity and mortality associated with the metabolic syndrome. Diabetes Care 24: 683-689.

5. Langer A, Freeman MR, Josse RG, Steiner G, Armstrong PW (1991) Detection of silent myocardial ischemia in diabetes mellitus. Am J Cardiol 67: 1073-1078.

6. Vinik Al, Erbas $\mathrm{T}$ (2001) Recognizing and treating diabetic autonomic neuropathy. Cleve Clin J Med 68: 928-930, 932, 934-44.

7. Vinik Al, Maser RE, Ziegler D (2011) Autonomic imbalance: prophet of doom or scope for hope? Diabet Med 28: 643-651.

8. Garruti G1, Giampetruzzi F, Vita MG, Pellegrini F, Lagioia P, et al. (2012) Links between metabolic syndrome and cardiovascular autonomic dysfunction. Exp Diabetes Res 2012: 615835.

9. Low PA, Benrud-Larson LM, Sletten DM, Opfer-Gehrking TL, Weigand SD, et al. (2004) Autonomic symptoms and diabetic neuropathy: a population-based study. Diabetes Care 27: 2942-2947.

10. Church TS, Thompson AM, Katzmarzyk PT, Sui X, Johannsen N, et al. (2009) Metabolic syndrome and diabetes, alone and in combination, as predictors of cardiovascular disease mortality among men. Diabetes Care 32: 1289-1294.

11. Vespasiani G, Bruni M, Meloncelli I, Clementi L, Amoretti R, et al. (1996) Validation of a computerised measurement system for guided routine evaluation of cardiovascular autonomic neuropathy Comput. Methods Programs Biomed 51: $211-216$

12. Portincasa $P$, Moschetta A, Berardino M, Di Ciaula A, Vacca M, et al. (2004) Impaired gallbladder motility and delayed orocecal transit contribute to pigment gallstone and biliary sludge formation in beta-thalassemia major adults. World J Gastroenterol 10: 2383-2390.

13. Cardone C, Bellavere F, Ferri M, Fedele D (1987) Autonomic mechanisms in the heart rate response to coughing. Clin Sci (Lond) 72: 55-60.

14. Cardone C, Paiusco P, Marchetti G, Burelli F, Feruglio M, et al. (1990) Cough test to assess cardiovascular autonomic reflexes in diabetes. Diabetes Care 13: $719-724$

15. Freeman R, Landsberg L, Young J (1999) The treatment of neurogenic orthostatic hypotension with 3,4-DL-threo- dihydroxyphenylserine: a randomized, placebo-controlled, crossover trial. Neurology 53: 2151- 2157.

16. Low PA, Walsh JC, Huang CY, McLeod JG (1975) The sympathetic nervous system in diabetic neuropathy. A clinical and pathological study. Brain 98 341-356.

17. Spallone V, Bellavere F, Scionti L, Maule S, Quadri R, et al. (2011) on behalf of the Diabetic Neuropathy Study Group of the Italian Society of Diabetology. Recommendations for the use of cardiovascular tests in diagnosing diabetic autonomic neuropathy. Nutr Metab Cardiovas Dis 21: 69-78.

18. Arunodaya GR, Taly AB (1995) Sympathetic skin response: a decade later. J Neurol Sci 129: 81-89.

19. Baum P, Petroff D, Classen J, Kiess W, Blüher S (2013) Dysfunction of autonomic nervous system in childhood obesity: a cross-sectional study. PLoS One 8: e54546.

20. Altomare DF, Portincasa P, Rinaldi M, Di Ciaula A, Martinelli E, et al. (1999) Slow-transit constipation: solitary symptom of a systemic gastrointestinal disease. Dis Colon Rectum 42: 231-240.

21. Portincasa P, Moschetta A, Berardino M, Di Ciaula A, Vacca M, et al. (2004) Impaired gallbladder motility and delayed orocecal transit contribute to pigment gallstone and biliary sludge formation in beta-thalassemia major adults. World J Gastroenterol 10: 2383-2390.

22. Heller PH, Perry F, Jewett DL, Levine JD (1990) Autonomic components of the human pupillary light reflex. Invest Ophthalmol Vis Sci 31: 156-162.

23. Freeman R (2006) Assessment of cardiovascular autonomic function. Clin Neurophysiol 117: 716-730.

24. Töyry JP, Niskanen LK, Mäntysaari MJ, Länsimies EA, Haffner SM, et al. (1997) Do high proinsulin and C-peptide levels play a role in autonomic nervous dysfunction?: Power spectral analysis in patients with non-insulin-dependent diabetes and nondiabetic subjects. Circulation 96: 1185-1191.

25. [No authors listed] (1994) Microvascular and acute complications in IDDM patients: the EURODIAB IDDM Complications Study. Diabetologia 37: 278285.

26. Neil H AW, Thompson AV, John S, McCarthy ST, Mann JI (1989) Diabetic autonomic neuropathy: the prevalence of impaired heart rate variability in a geographically defined Population. Diabetic Medicine 6: 20-24.

27. Takayama S, Sakura H, Katsumori K, Wasada T, Iwamoto Y (2001) A possible involvement of parasympathetic neuropathy on insulin resistance in patients with type 2 diabetes. Diabetes Care 24: 968-969.

28. [No authors listed] (1993) The effect of intensive treatment of diabetes on the development and progression of long-term complications in insulin-dependen diabetes mellitus. The Diabetes Control and Complications Trial Research Group. N Engl J Med 329: 977-986

29. Burger AJ, Weinrauch LA, D'Elia JA, Aronson D (1999) Effect of glycemic control on heart rate variability in type I diabetic patients with cardiac autonomic neuropathy. Am J Cardiol 84: 687-691.

30. Gaede P, Vedel P, Parving HH, Pedersen O (1999) Intensified multifactoria intervention in patients with type 2 diabetes mellitus and microalbuminuria: the Steno type 2 randomised study. Lancet 353: 617-622.

31. Shaw JE, Zimmet PZ, Gries FZ, Ziegler D (2003) Epidemiology of diabetic neuropathy. In: Griesw FA, Cameron NE, Low PA, and Ziegler D, (eds) Textbook of Diabetic Neuropathy, Thieme Medical Publishers, New York, NY, USA.

32. Vinik A, Erbas T, Casellini CM (2013) Diabetic cardiac autonomic neuropathy, inflammation and cardiovascular disease. J Diabetes Investig 4: 4-18.

33. Kim C, Pop-Busui R, Braffett B, Cleary PA, Bebu I, et al. (2015) Testosterone Concentrations and Cardiovascular Autonomic Neuropathy in Men with Type 
1 Diabetes in the Epidemiology of Diabetes Interventions and Complications Study (EDIC). J Sex Med 11: 2153-2159.

34. Szelag, B, Wroblewski M, Castenfors J, Henricsson M, Berntorp K, et al. (1999) Obesity, microalbuminuria, hyperinsulinemia, and increased plasminogen activator inhibitor 1 activity associated with parasympathetic neuropathy in type 2 diabetes. Diabetes Care 22: 1907-1908.

35. Herder C, Lankisch M, Ziegler D, Rathmann W, Koenig W, et al. (2009) Subclinical inflammation and diabetic polyneuropathy: MONICA/KORA Survey F3 (Augsburg, Germany). Diabetes Care 32: 680- 682.

36. Lampert R, Bremner JD, Su S, Miller A, Lee F, et al. (2008) Decreased heart rate variability is associated with higher levels of inflammation in middle-aged men. Am Heart J 156: 759.

37. Mohamed-Ali V, Flower L, Sethi J, Hotamisligil G, Gray R, et al. (2001) betaAdrenergic regulation of IL-6 release from adipose tissue: in vivo and in vitro studies. J Clin Endocrinol Metab 86: 5864-5869.

38. Borovikova LV, Ivanova S, Zhang M, Yang H, Botchkina GI, et al. (2000) Vagus nerve stimulation attenuates the systemic inflammatory response to endotoxin Nature 405: 458-462.

39. Margetic S, Gazzola C, Pegg GG, Hill RA (2002) Leptin: a review of its peripheral actions and interactions. Int J Obes Relat Metab Disord 26: 14071433.

40. Bado A, Levasseur S, Attoub S, Kermorgant S, Laigneau JP, et al. (1998) The stomach is a source of leptin. Nature 394: 790-793.

41. Ciriello J, Moreau JM (2013) Systemic administration of leptin potentiates the response of neurons in the nucleus of the solitary tract to chemoreceptor activation in the rat Neuroscience 229: 88-99.

42. da Costa Goncalves AC, Tank J, Diedrich A, Hilzendeger A, Plehm R, et al. (2009) Diabetic hypertensive leptin receptor-deficient $\mathrm{db} / \mathrm{db}$ mice develop cardioregulatory autonomic dysfunction. Hypertension 53: 387- 392.

43. Paolisso G, Manzella D, Montano N, Gambardella A, Varricchio M (2000) Plasma leptin concentrations and cardiac autonomic nervous system in healthy subjects with different body weights. J Clin Endocrinol Metab 85: 1810-1814.

44. Carlyle M, Jones OB, Kuo JJ, Hall JE (2002) Chronic cardiovascular and renal actions of leptin: role of adrenergic activity. Hypertension 39: 496-501.

45. Kreier F, Fliers E, Voshol PJ, Van Eden CG, Havekes LM, et al. (2002) Selective parasympathetic innervation of subcutaneous and intra-abdominal fat--functional implications. J Clin Invest 110: 1243-1250.

46. Hoyda TD, Samson WK, Ferguson AV (2009) Adiponectin depolarizes parvocellular paraventricular nucleus neurons controlling neuroendocrine and autonomic function. Endocrinology 150: 832- 840.

47. Tanida M, Shen J, Horii Y, Matsuda M, Kihara S, et al. (2007) Effects of adiponectin on the renal sympathetic nerve activity and blood pressure in rats. Exp Biol Med (Maywood) 232: 390-397.
48. Imai J, Katagiri H, Yamada T, Ishigaki Y, Ogihara T, et al. (2006) Cold exposure suppresses serum adiponectin levels through sympathetic nerve activation in mice. Obesity (Silver Spring) 14: 1132-1141

49. Wakabayashi S, Aso Y (2004) Adiponectin concentrations in sera from patients with type 2 diabetes are negatively associated with sympathovagal balance as evaluated by power spectral analysis of heart rate variation. Diabetes Care 27: 2392- 2397.

50. Smith AG, Russell J, Feldman EL, Goldstein J, Peltier A, et al. (2006) Lifestyle intervention for pre-diabetic neuropathy. Diabetes Care 29: 1294-1299.

51. Grandinetti A, Chow DC, Sletten DM, Oyama JK, Theriault AG, et al. (2007) Impaired glucose tolerance is associated with postganglionic sudomotor impairment. Clin Auton Res 17: 231-233.

52. Isak B, Oflazoglu B, Tanridag T, Yitmen I, Us O (2008) Evaluation of periphera and autonomic neuropathy among patients with newly diagnosed impaired glucose tolerance. Diabetes Metab Res Rev 24: 563-569.

53. Petrova M, Townsend R, Teff K L (2006) Prolonged (48-h) modest hyperinsulinemia decreases nocturnal heart rate variability and attenuates the nocturnal decrease in blood pressure in lean, normotensive humans. J. Clin. Endocrinol. Metab 91: 851-859.

54. Ziegler D, Zentai C, Perz S, Rathmann W, Haastert B, et al. (2006) Selective contribution of diabetes and other cardiovascular risk factors to cardiac autonomic dysfunction in the general population. Exp Clin Endocrinol Diabetes 114: 153-159.

55. Licht CM, Vreeburg SA, van Reedt Dortland AK, Giltay EJ, Hoogendijk WJ, et al. (2010) Increased sympathetic and decreased parasympathetic activity rather than changes in hypothalamica "pituitarya" adrenal axis activity is associated with metabolic abnormalities. J Clin Endocrinol Metab 95: 2458-2466.

56. Chang CJ, Yang YC, Lu FH, Lin TS, Chen JJ, et al. (2010) Altered cardiac autonomic function may precede insulin resistance in metabolic syndrome. Am J Med 123: 432-438.

57. Carnethon MR, Jacobs DR Jr, Sidney S, Liu K; CARDIA study (2003) Influence of autonomic nervous system dysfunction on the development of type 2 diabetes: the CARDIA study. Diabetes Care 26: 3035-3041.

58. Esposito K, Marfella R, Ciotola M, Di Palo C, Giugliano F, et al. (2004) Effect of a mediterranean-style diet on endothelial dysfunction and markers of vascular inflammation in the metabolic syndrome: a randomized trial. JAMA 292: 14401446

59. Carnethon MR, Prineas RJ, Temprosa M, Zhang ZM, Uwaifo G, et al. (2006) The association among autonomic nervous system function, incident diabetes, and intervention arm in the Diabetes Prevention Program. Diabetes Care 29: 914- 919.

60. Michalsen A, Knoblauch NT, Lehmann N, Grossman P, Kerkhoff G, et al. (2006) Effects of lifestyle modification on the progression of coronary atherosclerosis, autonomic function, and anginaa"the role of GNB3 C825T polymorphism. Am. Heart J 151: 870-877. 